\title{
FUSION OF CLASSIFIERS BASED ON A NOVEL 2-STAGE MODEL
}

\section{TIEN THANH NGUYEN ${ }^{1}$, ALAN WEE-CHUNG LIEW ${ }^{1}$, MINH TOAN TRAN ${ }^{2}$, THI THU THUY NGUYEN ${ }^{3}$, MAI PHUONG NGUYEN ${ }^{4}$}

\author{
${ }^{1}$ School of Information Technology, Griffith University, Australia. \\ ${ }^{2}$ School of Applied Mathematics and Informatics, Hanoi University of Science and Technology, Vietnam \\ ${ }^{3}$ College of Economics - Hue University, Vietnam \\ ${ }^{4}$ College of Business, Massey University, New Zealand \\ Email:tienthanh.nguyen2@griffithuni.edu.au
}

\begin{abstract}
:
The paper introduces a novel 2 -Stage model for multiclassifier system. Instead of gathering posterior probabilities resulted from base classifiers into Level1 data like in the original 2-Stage model, here we separate data in $K$ Level1 matrices corresponding to the $K$ base classifiers. These data matrices, in turn, are classified in sequence by a new classifier at the second stage to generate Level2 data. Next, Weight Matrix is proposed to combine Level2 data and predict label of observations in test set. Experimental results on CLEF2009 medical image database demonstrate the benefit of our model in comparison with several ensemble learning models.
\end{abstract}

Keywords:

Multi classifier system, combining classifiers, classifier fusion, Stacking algorithm.

\section{Introduction}

Combining classifiers to achieve low error rate has received much attention in recent years. Many state-of-art algorithms had been introduced and have been applied successfully to a variety of data sources. However, there is no one best combining algorithm. One algorithm may outperform others in some datasets but underperform in others.

Duin [1] summarized six strategies to build a combining system with good performance. The objective of these strategies is that "the more diverse the training set, base classifier and feature set, the better the performance of the ensemble system". The idea behind this approach is to decrease the correlation between input training sets, between base classifiers, and between feature sets. The six strategies include (a) different initializations, (b) different parameter choices, (c) different architectures, (d) different classifiers, (e) different training sets, and (f) different feature sets.

Here we focus on strategy (d). It is suggested that the base classifiers should come from significantly different approaches. The training set is shared among all base classifiers to generate posterior probability corresponding to each class and classifier. Ting [5] developed Stacking algorithm where the training set is divided to nearly equal disjoined B part. Each plays as test set once while the others plays as training set so all observations in the original set are tested once. Output of Stacking is the posterior probability that each observation belongs to a class according to each classifier. The set of posterior probability of all observations is called Level1 data, and Level0 data refers to the original data.

Let us denote the set of classes by $\left\{W_{j}\right\}$. Kuncheva [3] summarized three output types of a classifier of an observation $X$ as:

- Crisp Label: return only class label $P\left(W_{j} \mid X\right) \in\{0,1\}$ and $\sum_{j} P\left(W_{j} \mid X\right)=1$

- Fuzzy Label: return posterior probabilities that $X$ belongs to classes $P\left(W_{j} \mid X\right) \in[0,1] \quad$ and $\sum_{j} P\left(W_{j} \mid X\right)=1$

- Possibilistic Label: the same as Fuzzy Label but does not require the sum of all posterior probabilities to equal to one $P\left(W_{j} \mid X\right) \in[0,1] P\left(W_{j} \mid X\right)>0$

According to the above definition, Fuzzy Label is more applicable in our study since each posterior probability component reflects the support of a class to an observation. Note that possibilistic label can be transformed to Fuzzy Label by normalizing $P\left(W_{i} \mid X\right) / \sum_{j} P\left(W_{j} \mid X\right)$. Crisp Label also plays an important role in several algorithms [5][7]. In this paper, we focus on employing both Fuzzy Label and Crisp Label in the proposed algorithm.

We denote $N$ as number of observations, $K$ as number of base classifiers and $M$ as number of classes. For an observation $X_{i}, \quad P_{k}\left(\mathrm{~W}_{j} \mid X_{i}\right)$ is the probability that $X_{i}$ belongs to class $\mathrm{W}_{j}$ given by $k^{\text {th }}$ classifier. Level1 data of all 
observations, a $N \times M K$-posterior probability matrix is given by:

$$
\left[\begin{array}{lll}
P_{1}\left(\mathrm{~W}_{1} \mid X_{1}\right) \ldots P_{1}\left(\mathrm{~W}_{M} \mid X_{1}\right) & P_{2}\left(\mathrm{~W}_{1} \mid X_{1}\right) \ldots P_{2}\left(\mathrm{~W}_{M} \mid X_{1}\right) \ldots P_{K}\left(\mathrm{~W}_{1} \mid X_{1}\right) \ldots P_{K}\left(\mathrm{~W}_{M} \mid X_{1}\right) \\
P_{1}\left(\mathrm{~W}_{1} \mid X_{2}\right) \ldots P_{1}\left(\mathrm{~W}_{M} \mid X_{2}\right) & P_{2}\left(\mathrm{~W}_{1} \mid X_{2}\right) \ldots & P_{2}\left(\mathrm{~W}_{M} \mid X_{2}\right) \ldots P_{K}\left(\mathrm{~W}_{1} \mid X_{2}\right) \ldots P_{K}\left(\mathrm{~W}_{M} \mid X_{2}\right) \\
\ldots & \ldots & \\
P_{1}\left(\mathrm{~W}_{1} \mid X_{N}\right) \ldots P_{1}\left(\mathrm{~W}_{M} \mid X_{N}\right) & P_{2}\left(\mathrm{~W}_{1} \mid X_{N}\right) \ldots P_{2}\left(\mathrm{~W}_{M} \mid X_{N}\right) \ldots P_{K}\left(\mathrm{~W}_{1} \mid X_{N}\right) \ldots P_{K}\left(\mathrm{~W}_{M} \mid X_{N}\right)
\end{array}\right]
$$

whereas Level1 data of $X_{i}$ is defined:

$$
\operatorname{Level1}\left(X_{i}\right)_{i=1, N} \square\left[\begin{array}{ccc}
P_{1}\left(\mathrm{~W}_{1} \mid X_{i}\right) & \ldots & P_{1}\left(\mathrm{~W}_{M} \mid X_{i}\right) \\
\vdots & \ddots & \vdots \\
P_{K}\left(\mathrm{~W}_{1} \mid X_{i}\right) & \cdots & P_{K}\left(\mathrm{~W}_{M} \mid X_{i}\right)
\end{array}\right]
$$

** symbol in (2) has error.

Kittler [6] presented six combining rules namely Sum, Product, Vote, Min, Max, and Average. These Rules are simple in calculation and in several datasets they reach lower error rate than that of the base classifiers. Ting [5] proposed Multiple Response Linear Regression algorithm (MLR) to combine posterior probabilities of each observation based on sum of weights calculated from $\mathrm{K}$ linear regression functions. Kuncheva [3] applied Fuzzy Relation to find the relationship between posterior probability matrix (2) and Decision Template for each class computed on posterior probability of observations and its true class label. Metz [7] combined Staking, Correspondence Analysis and $\mathrm{KNN}$ in a single algorithm called SCANN. His idea was to find the relationship between rows (include all observations) and columns (include classification results of all classifiers). Todorovski proposed Meta Decision Tree [8] [9], a Decision Tree on Level1 data. It is different to the ordinary Decision Tree as at each node it chooses a classifier to classify instead of selecting a value to split.

Besides, a coarse 2-Stage approach was introduced by Benediktsson [10] in which he uses two base classifiers, namely a Neural Network (NN) classifier and a Gaussian Maximum Likelihood (GML) classifier in the first stage and another NN classifier in the second stage. If both base classifiers agree, observation is classified to their agreed class. If they do not agree, the observation is rejected. All rejected observations are collected and classify with a second NN in the second stage. This approach reached good results with several datasets although it is not general enough in most situations.

A 2-Stage combining model was published in [11] in which $\mathrm{K}$ different feature vectors extracted from an object is classified by $\mathrm{K}$ base classifiers respectively. However, extracting more than one feature vector is not always possible in most situations. In this case, one can use Stacking. In the first stage, a dataset is classified by $K$ base classifiers though Stacking to obtain the Level1 data. Next, the Level1 data is classified by a new classifier (denoted by C). This kind of model has two benefits. First, Level1 data usually has low dimensions compared with Level0 data, for instance, the $D$-feature vector of an image (Level0 data) may have more dimension than that of Level1 data, $D>M \times K$ [15]. Moreover, Level1 data can be viewed as a "scaled result" in $[0,1]^{M K}$ by $K$ base classifiers so it would have more discrimination ability than Level0 data.

In this work, we propose a new 2-Stage model that has better performance than the original 2-Stage model of [11]. In Section 2, we introduce the architect of our 2-Stage model and present one combining algorithm based on weights. Experimental evaluations are conducted on CLEF 2009 medical image database using the proposed model. Finally, we summarize and propose several possible developments for our model.

\section{Proposed Model}

In the original 2-Stage model, the posterior probability results from base classifiers are grouped together into a single vector and this vector becomes the feature vector for $\mathrm{C}$. In our approach, we separate the posterior probability results into $\mathrm{K}$ Level1 data correspond to the $\mathrm{K}$ base classifiers, which are then classified by $\mathrm{C}$ in the second stage. After that, the $\mathrm{K}$ posterior probability results given by $\mathrm{C}$ are combined to give the final classification. Our idea is based on Strategy (e) in Section 1 where K Level1 data can be viewed as K different training sets. Our objective here is to increase the diversity so as to decrease the correlation between input training sets. The proposed model is illustrated in Figure 1.

In our model, Level1(k) of all observations from the $k^{\text {th }}$ classifier is a $N \times M-$ posterior probability matrix $\left\{P_{k}\left(\mathrm{~W}_{j} \mid X_{i}\right)\right\}$ (3). After classified by C, Level2(k) is also a $N \times M$ - posterior probability matrix $\left\{P_{k}^{C}\left(\mathrm{~W}_{j} \mid X_{i}\right)\right\} j=\overline{1, M}$ $i=\overline{1, N}$ (4). 


$$
\begin{aligned}
& \underset{k=1, K}{L e v e l 1(k)} \square {\left[\begin{array}{l}
P_{k}\left(\mathrm{~W}_{1} \mid X_{1}\right) P_{k}\left(\mathrm{~W}_{2} \mid X_{1}\right) \ldots P_{k}\left(\mathrm{~W}_{M} \mid X_{1}\right) \\
P_{k}\left(\mathrm{~W}_{1} \mid X_{2}\right) P_{k}\left(\mathrm{~W}_{2} \mid X_{2}\right) \ldots P_{k}\left(\mathrm{~W}_{M} \mid X_{2}\right) \\
\ldots \\
P_{k}\left(\mathrm{~W}_{1} \mid X_{N}\right) P_{k}\left(\mathrm{~W}_{2} \mid X_{N}\right) \ldots P_{k}\left(\mathrm{~W}_{M} \mid X_{N}\right)
\end{array}\right] } \\
& \underset{k=1, K}{\text { Level2 }(k) \square}\left[\begin{array}{l}
P_{k}^{C}\left(\mathrm{~W}_{1} \mid X_{1}\right) P_{k}^{C}\left(\mathrm{~W}_{2} \mid X_{1}\right) \ldots P_{k}^{C}\left(\mathrm{~W}_{M} \mid X_{1}\right) \\
P_{k}^{C}\left(\mathrm{~W}_{1} \mid X_{2}\right) P_{k}^{C}\left(\mathrm{~W}_{2} \mid X_{2}\right) \ldots P_{k}^{C}\left(\mathrm{~W}_{M} \mid X_{2}\right) \\
\ldots \\
P_{k}^{C}\left(\mathrm{~W}_{1} \mid X_{N}\right) P_{k}^{C}\left(\mathrm{~W}_{2} \mid X_{N}\right) \ldots P_{k}^{C}\left(\mathrm{~W}_{M} \mid X_{N}\right)
\end{array}\right]
\end{aligned}
$$

while Level2 data of individual observation $X$ is defined by:

$$
\text { Level2 }(X) \square\left[\begin{array}{ccc}
P_{1}^{C}\left(\mathrm{~W}_{1} \mid X\right) & \ldots & P_{1}^{C}\left(\mathrm{~W}_{M} \mid X\right) \\
\vdots & \ddots & \vdots \\
P_{K}^{C}\left(\mathrm{~W}_{1} \mid X\right) & \cdots & P_{K}^{C}\left(\mathrm{~W}_{M} \mid X\right)
\end{array}\right]
$$

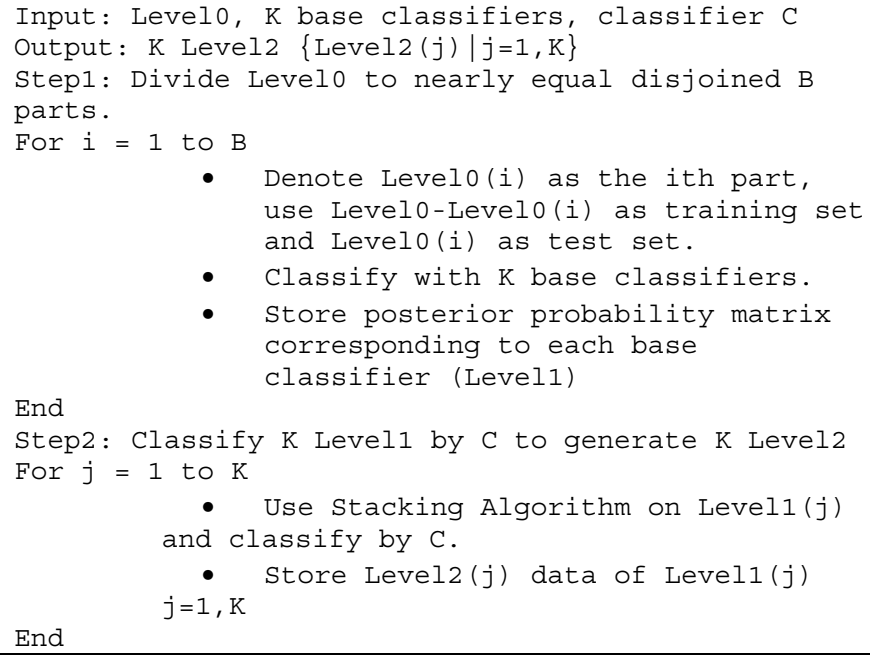

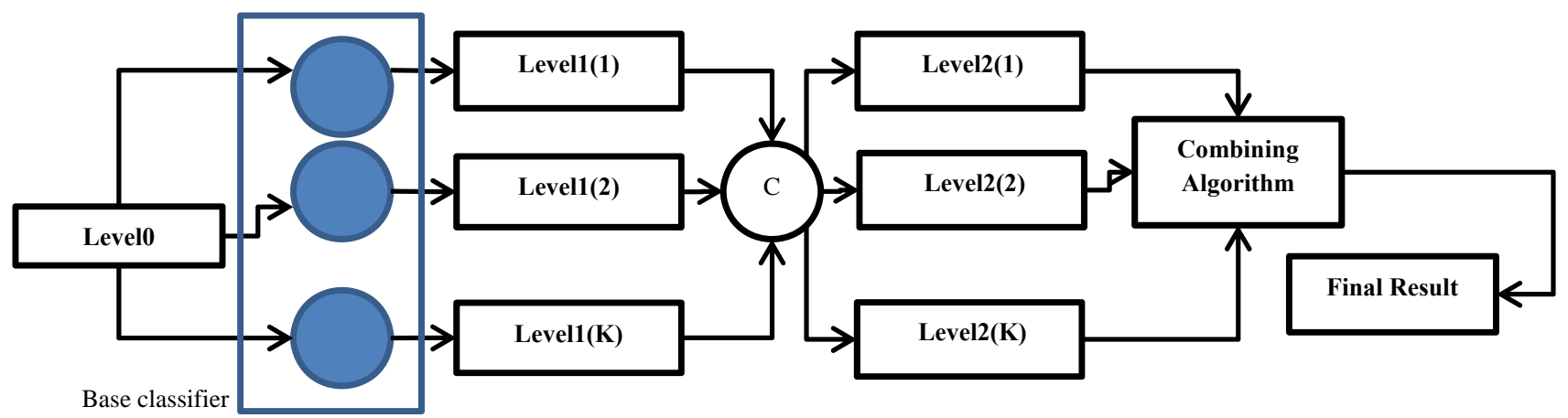

Figure 1. The proposed 2-Stage model

\section{Combining Algorithm}

It is reasonable to assume that the contribution of each base classifier on combining result would not be the same since some base classifiers would be better suited to the classification problem and would contribute more to the combining result. Hence, we propose one combining algorithm where the weights of base classifiers on $\mathrm{C}$ are different. Here we are inspired by the idea of MLR introduced by Ting [5].

Our idea is to expand the Sum rule [6] by building weights as a $K \times M$ matrix $\boldsymbol{\Psi}=\left\{\omega_{k j}\right\}^{T}$ in which $k^{\text {th }}$ base classifier put a weight $\omega_{k j}$ on C corresponding to $j^{\text {th }}$ class. We only impose a condition that all weights be non-negative $\omega_{k j}>0 \forall k, j$. Performing combination task based on $\boldsymbol{\Psi}$, we have $\mathrm{M}$ sums for $X$ according to the $M$ classes:

$$
\text { class } j^{\text {th }} \leftarrow \sum_{k=1}^{K} \omega_{k j} P_{k}^{C}\left(\mathrm{~W}_{m} \mid X\right) \quad j=\overline{1, M}
$$

Here, $m^{\text {th }}$ column vector of $\boldsymbol{\Psi}$ associated with $m^{\text {th }}$ class is extracted: $\quad \mathbf{W}_{m}=\left\{\omega_{k m}\right\}^{T} \quad k=\overline{1, K}$ for each $m=\overline{1, M}$. $N \times K$ Level2 posterior probability matrix of $m^{\text {th }}$ class is given by: 


$$
\mathbf{P}_{m}=\left(\begin{array}{ccc}
P_{1}^{C}\left(W_{m} \mid X_{1}\right) & \ldots & P_{K}^{C}\left(W_{m} \mid X_{1}\right) \\
\vdots & \ddots & \vdots \\
P_{1}^{C}\left(W_{m} \mid X_{N}\right) & \cdots & P_{K}^{C}\left(W_{m} \mid X_{N}\right)
\end{array}\right)
$$

Let the label vector be:

$$
\mathbf{Y}_{m}=\left\{y_{m}\left(X_{i}\right)\right\}^{T} \quad i=\overline{1, N}
$$

where $y_{m}\left(X_{i}\right)$ is Crisp Label of $X_{i}$ corresponding to $m^{\text {th }}$ class.

$$
y_{m}\left(X_{i}\right)=\left\{\begin{array}{l}
1 \text { if } X_{i} \in \mathrm{W}_{m} \\
0 \text { otherwise }
\end{array}\right.
$$

Our objective is to minimize the distance between sum (6) and true class label of all observation in training set. So from (7) and (8) we have a system of equations:

$$
\mathbf{P}_{m} \mathbf{W}_{m}=\mathbf{Y}_{m}
$$

For $m=\overline{1, M}$, we have $M$ system of equations in total. Solving each of them individually by non-negative regression we will have all columns vectors of $\boldsymbol{\Psi}$

During testing, we compute $M$ sums based on $\boldsymbol{\Psi}$ :

$$
\sum_{k=1}^{K} \omega_{k j} P_{k}^{C}\left(\mathrm{~W}_{m} \mid X_{\text {test }}\right) \quad j=\overline{1, M}
$$

and class label of $X_{\text {test }}$ is predicted by:

$$
X_{\text {test }} \in W_{t} \text { if } t=\arg \max _{m=1, M}\left(\sum_{k=1}^{K} \omega_{k j} P_{k}^{C}\left(\mathrm{~W}_{m} \mid X_{\text {test }}\right)\right)
$$

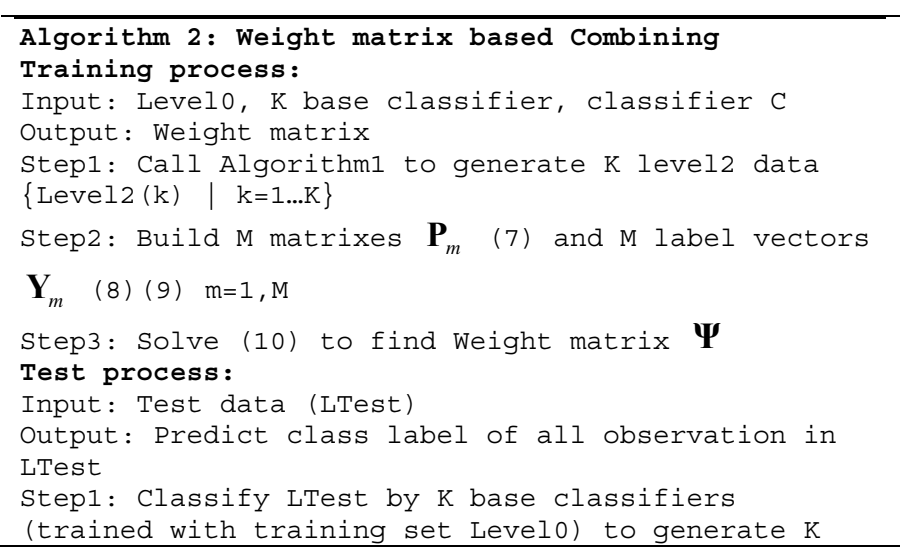

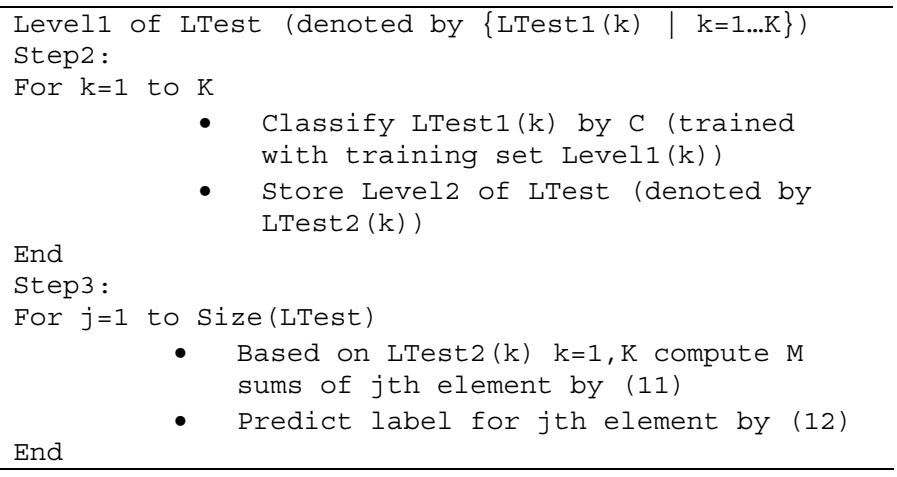

\section{$4 \quad$ Experimental Results}

We conducted experiment on CLEF 2009 database, a large set of medical image collected by Archen University. It includes 15,363 images allocated in 193 hierarchical categories. In our experiment we choose the first 7 classes and the entire 10 classes where each has different number of images (TABLE 1). First, we perform some pre-processing like histogram equation and then, feature vector is extracted. Here LDA, QDA and Naïve Bayes are chosen as base classifiers and $\mathrm{C}$ is $\mathrm{KNN}$ (with $\mathrm{K}$ set to 5, denoted as KNN(5)). Histogram of Local Binary Pattern (HLBP) [15] is extracted as feature. The results of the experiment are summarized in TABLE 2 and 3.

We performed 10 -fold cross validation by dividing the dataset into 10 nearly equal disjoined parts. Each part plays as a test set once while the others play as the training set. To ensure objectiveness, we run the test 10 times so we have a total of 100 test outcomes. Statistical Toolbox in Matlab2013a is used to develop our model. In our assessment, we compare the error rates of 4 approaches: best result from base classifiers, Direct Model (classify by $\mathrm{KNN}(5)$ ) (**what is direct model?), original 2-Stage model, and proposed model. For comparison purpose, we use one tail statistical test to compare two expectations (parameter $\alpha$ is set by 0.05)

TABLE 1. CLEF 2009 CLASS INFORMATION USED IN EXPERIMENT

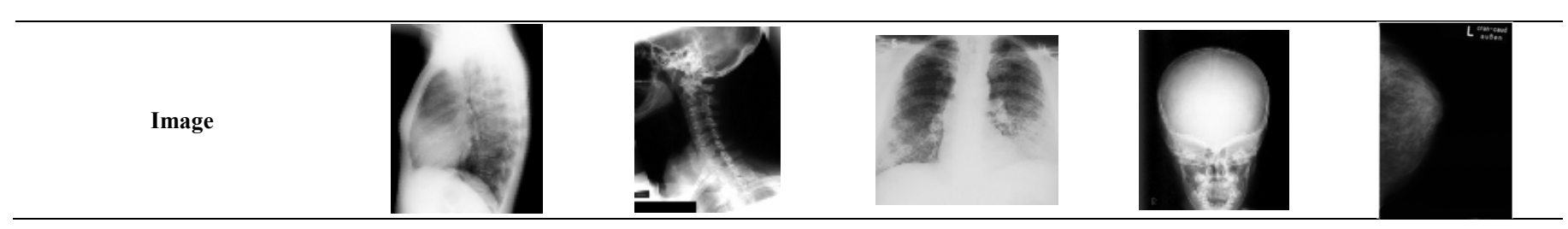


2014 International Conference on Machine Learning and Cybernetics, ICMLC2014, Lanzhou, China, 1316 July 2014

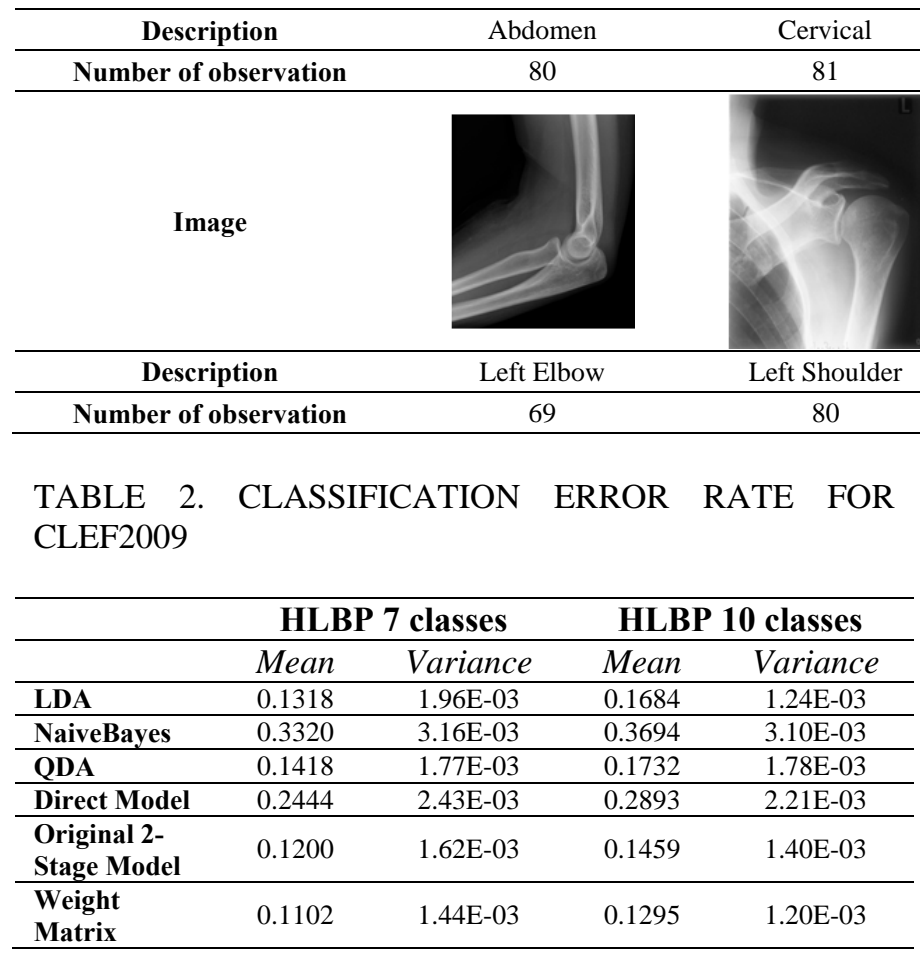

TABLE 3. STATISTICAL TEST RESULT BETWEEN OUR COMBINING ALGORITHM AND COMPETITORS (**for 7 or 10 class?)

\begin{tabular}{lccc}
\hline & Better & Competitive & Worse \\
\hline Weight Matrix vs. Select Best & 2 & 0 & 0 \\
\hline $\begin{array}{l}\text { Weight Matrix vs. Direct Model } \\
\text { by KNN }\end{array}$ & 2 & 0 & 0 \\
\hline $\begin{array}{l}\text { Weight Matrix vs. Original 2- } \\
\text { Stage Model }\end{array}$ & 2 & 0 & 0 \\
\hline
\end{tabular}

It is evident that our approach compared favorably with Direct Model by $\mathrm{KNN}(5)$ and original 2-Stage model (TABLE 3). Weight based algorithm achieves lowest error (11\% and 13\%), followed by original 2-Stage model (12\% and $14.59 \%)$, Select Best (13.18\% and 16.84\%) and Direct Model (24.44\% and 28.93\%) (TABLE 2).

To sum up, Weight-based combining algorithm outperforms Direct Model, original 2-Stage mode and best result from base classifiers. However, Level2 data of test set as well as that of original Level0 data need to be generated in the Weight-based algorithm, as a result, computation cost increases.

5 Conclusion

\section{References}

[1] Robert P. W. Duin, The Combining Classifier: To Train or Not to Train? Proceedings. 16th International Conference on Pattern Recognition, Volume 2,pp. 765-770, 2002.

[2] Alan Julian Izenman, Modern Multivariate Statistical Techniques, Chapter 14, Springer.

[3] L. I. Kuncheva, James C. Bezdek and Robert P. W. Duin, Decision Templates for Multi Classifier Fusion: An Experimental Comparison, Pattern Recognition, 34, (2),pp. 299-314, 2001.

[4] L. I. Kuncheva, A theoretical Study on Six Classifier Fusion Strategies, IEEE Transactions on Pattern Analysis and Machine Intelligence, Vol.24, No.2, February 2002.

[5] Kai Ming Ting, Ian H. Witten, Issues in Stacked Generation, Journal of Artificial In Intelligence Research 10, pp. 271-289, 1999.

[6] Josef Kittler, Mohamad Hatef, Robert P. W. Duin and Jiri Matas, On Combining Classifiers, IEEE Transactions on Pattern Analysis and Machine Intelligence, Vol.20, No.3, March 1998. 
2014 International Conference on Machine Learning and Cybernetics, ICMLC2014, Lanzhou, China, 1316 July 2014

[7] Christopher Merz, Using Correspondence Analysis to Combine Classifiers, Machine Learning 36, pp. 33-58, 1999.

[8] Ljupco Todorovski, Saso Džeroski, Combining Classifiers with Meta Decision Trees, Machine Learning 50, pp. 223-249, 2003.

[9] Saso Džeroski, Bernard Ženko, Is Combining Classifiers with Stacking Better than Selecting the Best One? Machine Learning 54, pp. 255-273, 2004.

[10] J. A. Benediktsson, I. Kanellopoulos, Classification of Multisource and Hyperspectral Data Based on Decision Fusion, IEEE Transactions on Geoscience and Remote Sensing, Vol. 37, No 3, May 1999.

[11] L. Lepisto, I. Kunttu, J. Autio and A. Visa, Classification of Non-Homogeneous Texture Images by Combining Classifier, Proceedings International Conference on Image Processing, Volume 1, pp. 981-984, 2003.

[12] Mehmet Umut Sen, Hakan Erdogan, Linear classifier combination and selection using group sparse regularization and hinge loss, Pattern Recognition Letters 34, pp. 265-274, 2013.

[13] Yong-Sheng Chen, Yi-Ping Hung, Ting-Fang Yen and Chiou-Shann Fuh, Fast and versatile algorithm for nearest neighbor search based on a lower bound tree, Pattern Recognition 40, pp. 360-375, 2007.

[14] Alexander K. Seeward, How to Make Stacking Better and Faster While Also Taking Care of an Unknown Weakness, Proceedings of the Nineteenth International Conference on Machine Learning, pp. 554-561, 2002.

[15] Byoung Chul Ko, Seong Hoon Kim and Jea Yeal Nam, X-ray Image Classification Using Random Forests with Local Wavelet-Based CS-Local Binary Pattern, J Digital Imaging, Vol. 24, pp. 1141-1151, 2011.

[16] UCI Machine Learning Repository, http://archive.ics.uci.edu/ml/datasets.html. 